\title{
L'énergie éolienne en Allemagne du sud-ouest - origines, problèmes et solutions à la transformation des paysages de la transition énergétique
}

Wind power in Southwest Germany - Causes, Problems and Solutions of a largescale transformation of landscapes in the energy transition

Windkraft in Südwest-Deutschland - Ursachen, Probleme und Lösungen einer großräumigen Umgestaltung von Landschaften in der Energiewende

Michael Altmoos

\section{(2) OpenEdition}

\section{Electronic version}

URL: http://journals.openedition.org/rge/5382

ISSN: 2108-6478

\section{Publisher}

Association des géographes de l'Est

Printed version

Date of publication: 1 January 2015

ISSN: 0035-3213

\section{Electronic reference}

Michael Altmoos, «L'énergie éolienne en Allemagne du sud-ouest - origines, problèmes et solutions à la transformation des paysages de la transition énergétique », Revue Géographique de l'Est [Online], vol. $55 / n^{\circ} 1-2$ | 2015, Online since 09 June 2015, connection on 08 September 2020. URL : http:// journals.openedition.org/rge/5382 


\section{L'énergie éolienne en Allemagne du sud-ouest - origines, problèmes et solutions à la transformation des paysages de la transition énergétique}

Wind power in Southwest Germany - Causes, Problems and Solutions of a largescale transformation of landscapes in the energy transition

Windkraft in Südwest-Deutschland - Ursachen, Probleme und Lösungen einer großräumigen Umgestaltung von Landschaften in der Energiewende

Michael Altmoos

Le Professeur Michel Deshaies (Géographie, Université de Lorraine) a traduit cet article de l'allemand en français.

\section{Introduction}

1 Depuis une quinzaine d'années on construit de grandes installations de production éolienne dans de nombreuses parties de l'Europe. Cette dynamique est particulièrement forte en Allemagne (Deshaies 2013), et dans une moindre mesure également dans certaines parties de la France. À travers toute l'Europe on encourage un développement encore plus important de l'éolien. D'après les statistiques disponibles, rien qu'en Allemagne il y avait en février 2014 déjà plus de 25000 installations et d'après les plans actuels, des dizaines de milliers d'autres devraient s'y ajouter. Cela devrait avoir un nouvel impact spatial avec des conséquences multiples qui vont être évoquées dans cet article. 
2 En ce qui concerne l'éolien, il faut distinguer les conditions entre les sites offshore et onshore ; dans cet article, il s'agit de sites onshore. Après une phase de développement précoce de petites éoliennes en Allemagne du Nord (moins de $150 \mathrm{~m}$ de haut), depuis environ cinq ans ce sont aussi les „Mittelgebirge“ du sud-ouest de l'Allemagne dans les Länder de Rhénanie-Palatinat, de la Sarre, de la Hesse, du Bade-Wurtemberg et d'une partie de la Rhénanie-du-Nord-Westphalie qui sont de plus en plus gagnés par l'implantation d'éoliennes. Le rythme de construction est particulièrement élevé en Rhénanie-Palatinat et dans la Sarre; ce qui a conduit à une forte densification des grandes éoliennes (plus de $150 \mathrm{~m}$ ), alors que relativement peu d'éoliennes ont été construites en Bade-Wurtemberg, dans une partie de la Hesse et en Bavière. Mais là aussi il existe actuellement une forte dynamique en matière d'éolien. À la différence des anciens petits moulins à vent qui s'intégraient dans le paysage, les „parcs éoliens“ terrestres dont il s'agit ici sont de nouvelles infrastructures aux dimensions horsnormes (Figure 1).

Dans le sud-ouest de l'Allemagne on peut particulièrement bien étudier les évolutions, les opportunités, mais aussi les problèmes qui se posent en France et dans d'autres parties de l'Europe. À l'échelle mondiale le développement de l'énergie éolienne représente une évolution majeure (par exemple aux USA ou en Chine).

Dans cet article on analysera d'abord à partir de la bibliographie et de nos observations les raisons et les facteurs (driving forces) qui sont responsables de ce développement. Puis on présentera une vue d'ensemble des problèmes spatiaux liés à l'énergie éolienne. Enfin, à partir de cela et suivant des perspectives géographiques on proposera des solutions pour réaliser à l'avenir des compromis acceptables entre l'homme, la nature et les besoins énergétiques 
Figure 1. Illustrations photographiques de la problématique
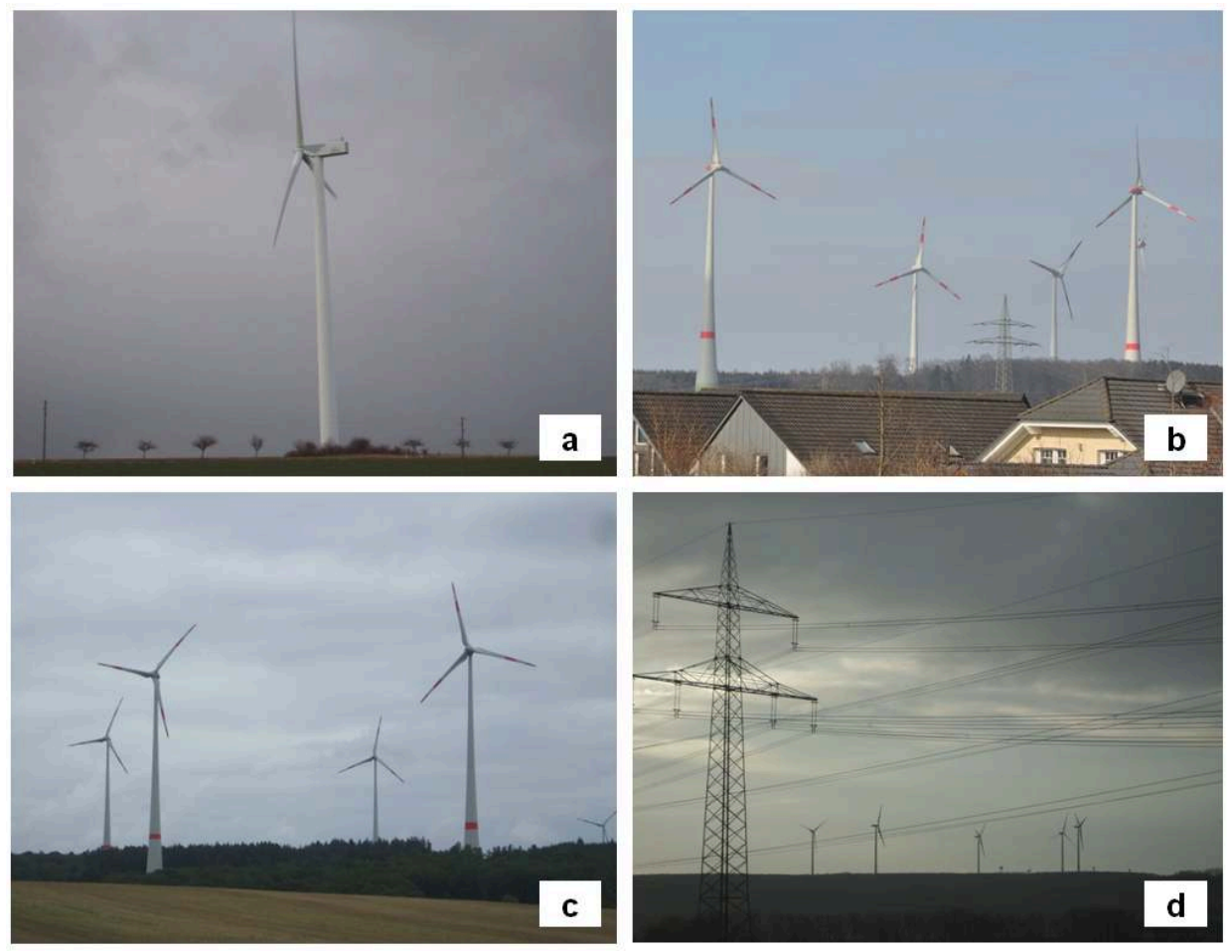

(a) De nouvelles éoliennes pour la plupart de plus de $200 \mathrm{~m}$ de haut, constituent de nouveaux marqueurs paysagers dominant tout. II n'y a jamais eu auparavant des objets de telles dimensions marquant autant l'espace. (b) Elles dominent les zones d'habitat rural en concentrations serrées et (c) elles sont aussi de plus en plus construites sous forme de parcs éoliens dans des forêts et des zones naturelles de valeur. (d) Il en résulte un paysage énergétique décentralisé et couvrant presque tout l'espace : un changement paysager extrême qui se propage à travers tous les types d'espaces en les nivelant, multipliant les problèmes (photos de Michael Altmoos, a et d de la région d'Alzey/ Rheinhessen, b et c autour de Kirchberg/Hunsrück - Rhénanie-Palatinat).

\section{Facteurs de développement de l'énergie éolienne}

\section{A. La "transition énergétique“: cadre politique et système de subventions (EEG)}

5 À l'origine du développement de l'énergie éolienne il y a les subventions aux énergies renouvelables qui se sont mises en place dans le contexte mondial de la mutation des systèmes énergétiques (Smil, 2010). Les raisons d'une transition énergétique sont semblables dans le monde entier; mais en Allemagne elles sont particulièrement mises en avant et justifiées de la manière suivante : (1) prévenir les risques (avec une insistance particulière sur les risques du nucléaire), (2) la consommation d'espace (par exemple des mines de lignite) et les diverses dégradations de la nature par les énergies fossiles, (3) le caractère fini des ressources énergétiques fossiles, (4) le point de vue géopolitique, en l'occurrence diminuer les dépendances vis-à-vis des fournisseurs d'énergie fossile et enfin (5) la protection du climat (éviter les émissions de dioxyde de carbone pour les besoins de la production d'énergie).

6 En Allemagne, la catastrophe de Fukushima (Tsunami au Japon accompagné d'un accident nucléaire en 2011) a été un tournant spectaculaire de la politique énergétique qualifiée de „Energiewende“ (littéralement tournant énergétique plutôt que 
transition). Même si depuis longtemps, les énergies renouvelables étaient dans une certaine mesure subventionnées, il fallait maintenant une sortie totale du nucléaire particulièrement rapide; ce qui supposait que les énergies renouvelables puissent, le plus vite possible, couvrir une part encore plus importante de l'approvisionnement énergétique. Pour cela, il existe actuellement un large consensus en Allemagne. Selon des sondages sur des échantillons représentatifs de la population, 90\% des Allemands sont favorables à cette „Energiewende“ et $80 \%$ sont favorables au développement de l'énergie éolienne ${ }^{1}$. Néanmoins, se développe aussi lentement une certaine résistance qui condamne cette nouvelle destruction de la nature, l'absence de concept viable et l'impasse économique ${ }^{2}$.

7 La production d'énergie nucléaire et en particulier le traitement des déchets et des dégâts environnementaux ont toujours été soutenus et subventionnés par les crédits publics. En matière énergétique il n'y a encore jamais eu de marché libre. Le fort subventionnement des énergies renouvelables marque ainsi un tournant dans les priorités, mais pas un changement fondamental dans la façon de penser. À côté de la biomasse et un peu de l'hydroélectricité, ce sont surtout l'énergie solaire et l'énergie éolienne qui en ont profité. Les éoliennes sont non seulement plus remarquables, mais ont aussi une place plus importante pour la production d'électricité que les installations solaires, elles-mêmes actuellement en plein développement.

En Allemagne, ce que l'on appelle la loi sur les énergies renouvelables (Erneuerbare Energien Gesetz: EEG) est au cœur même de cet essor. Le système complexe d'aide financière mis en place par la loi EEG fait que presque partout, l'installation et l'exploitation d'éoliennes rapporte suffisamment d'argent aux producteurs comme aux propriétaires des terrains et aux communes, même sur des sites économiquement non rentables. Il s'agit en l'occurrence d'argent public qui est détourné vers cet objectif. Le prix est bien visible pour les consommateurs d'électricité, alors que le soutien au charbon et à l'atome était autrefois intégré aux impôts généraux et était peu visible, contrairement au prix actuel payé pour les énergies renouvelables. Seuls les gros consommateurs industriels de courant électrique sont épargnés par cette tarification des énergies renouvelables, en raison d'exemptions fortement discutées. Aussi la loi EEG est-elle l'objet non seulement de discussions économiques et écologiques, mais aussi sociales. Le prix du courant électrique pour les petits consommateurs en Allemagne est ainsi plus élevé que presque partout en Europe, à l'exception du Danemark où l'énergie éolienne a été également fortement développée.

Une des conséquences de cette situation est que certains acteurs ont tout intérêt à planifier, construire et exploiter toujours plus d'éoliennes, ou à louer des terres pour cela. Ce sont en particulier de nouvelles firmes spécialisées, des propriétaires terriens et aussi des communes qui en conséquence poussent au développement. Et tout cela est de diverses manières soutenu politiquement. Il y a aussi de plus en plus de „parcs éoliens citoyens" dans lesquels chacun peut investir avec la perspective de toucher une rente financière. En fait, il est apparu que les revenus réels étaient souvent bien en deçà des rentes espérées et qu'il pouvait même y avoir des pertes ${ }^{3}$. Cependant, l'intérêt financier est grand et constitue la principale motivation pour beaucoup de citoyens d'investir dans l'énergie éolienne, notamment dans les espaces ruraux en difficultés.

C'est ainsi qu'un système de subventions voulu par le politique est devenu un facteur déterminant d'une transformation du paysage. Sous d'autres formes, il y a déjà eu souvent des changements paysagers consécutifs à des systèmes de subventions (par 
exemple les réformes agraires), comme l'illustre de multiples façons l'histoire des paysages de l'Europe centrale (par exemple Küster 1995, Schaich \& Konold 2011); mais avec l'énergie éolienne la vitesse et les dimensions de ces transformations sont extraordinaires.

En tant qu'élément central pour le développement de l'éolien, la loi EEG a été modifiée par la réforme entrée en vigueur le 1er août 2014, suite aux nombreuses critiques formulées. Les derniers changements introduits par la réforme concernent surtout une diminution des tarifs en faveur des installations solaires et de biomasse, un peu moins l'éolien. Les tarifs d'achat de l'éolien ont été un peu abaissés en 2014 et on a fixé un "corridor" annuel de 2400 à $2600 \mathrm{MW}$ pour les nouvelles installations; ce qui ne devrait pas beaucoup ralentir le développement actuel très dynamique. Pourtant, la loi EGG est encore en discussion et au centre des intérêts divergents des acteurs; l'industrie éolienne ayant largement réussi à imposer ses vues et profitant encore de la situation (état en septembre 2014). La loi EEG a été ainsi jusqu'à présent la principale force d'impulsion qui a conduit à une transformation totale des espaces et qui doit en conséquence encore longtemps retenir notre attention.

\section{B. Planifier et penser à l'échelle locale}

Un facteur important pour l'orientation du développement de l'éolien est de mettre en place une planification basée sur les considérations géographiques (Gailing \& Leibenath 2013). Dans tous les Länder allemands il existe une „planification du paysage“ à différentes échelles. Elle demande un regroupement des éoliennes et des distances à respecter par rapport aux zones habitées.

13 Cependant on a tendance traditionnellement à penser à une échelle trop locale. Les écarts entre les sites d'implantation sont trop faibles et les dimensions des nouvelles éoliennes (plus de $200 \mathrm{~m}$ de haut, plus de 50 ha pour les parcs éoliens) sont inadaptées. Le facteur pensé à l'échelle locale est essentiel pour comprendre les évolutions dans le sud-ouest de l'Allemagne. Dans beaucoup de régions, il y a eu une occupation presque complète de l'espace, par exemple dans le Hunsrück oriental et dans la Hesse rhénane (Rhénanie-Palatinat, figure 1). Mais même dans les espaces restés relativement libres d'éoliennes, il est prévu un fort développement (par exemple dans le parc naturel Soonwald-Nahe, figure 2). Les écarts entre les parcs éoliens sont souvent inférieurs à 10-15 km et les éoliennes sont à moins d'un km par rapport aux lieux habités; parfois directement en bordure d'agglomération. Pour conserver des paysages libres d'éoliennes il faudrait que les écarts entre les parcs soient nettement plus grands (plus de $20-30 \mathrm{~km})$.

14 Là où il y avait autrefois de grandes orientations pour la planification, celles-ci ont été mises de côté à cause des forces poussant au développement de l'éolien. Les éoliennes sont aussi installées dans des espaces protégés (parcs naturels, zones de protection des paysages et zones Natura 2000) qui ne sont plus considérées comme devant être épargnées. En Rhénanie-Palatinat, en Sarre et en Bade-Wurtemberg la responsabilité de la planification a été transmise à l'échelle communale; en Hesse elle est sur le point de l'être. La conséquence est que maintenant presque chaque commune dont le territoire est à $10-20 \mathrm{~km}$ de la prochaine voudrait avoir un parc éolien à cause des revenus financiers. Cette situation est justifiée par l'argument démocratique que ce sont ceux qui sont concernés sur place qui doivent décider. Pourtant, si ce principe démocratique 
paraît raisonnable, ce qu'il y a de particulier dans la situation c'est qu'en raison même des dimensions des installations et de l'exigence d'un grand „tournant énergétique“ il faut que l'énergie éolienne soit pensée dans un cadre spatial large, en contradiction avec la conception très locale qui prédomine actuellement en Allemagne du Sud-ouest.

\section{La motivation de la protection du climat}

Les énergies renouvelables ont le grand avantage d'émettre beaucoup moins de dioxyde de carbone que les centrales thermiques et présentent aussi nettement moins de risques et de problèmes que les centrales nucléaires. Elles sont considérées comme jouant un rôle central pour la protection du climat. Mais cela est très sommaire sur le plan technique et en fait insatisfaisant comme cela va être expliqué ci-dessous. Cependant, la protection du climat constitue un motif important pour le développement de l'énergie éolienne, en lien avec les gains venant du système mis en place par la loi EEG.

16 Ce sont donc les facteurs principaux du grand boom éolien qui marque de nombreux espaces. La combinaison entre la volonté politique et le soutien public pour les énergies renouvelables renforce les incitations économiques du système de subvention (loi EEG) qui favorisent la fragmentation particulièrement importante de la planification.

\section{Les Problèmes posés par l'énergie éolienne}

17 L'énergie éolienne offre des opportunités, mais pose aussi des problèmes dont on va présenter ici les grandes lignes afin de proposer les solutions possibles.

\section{A. Les effets sur les hommes}

Suivant les sites et les types d'installation, les hommes peuvent être gênés par le bruit, les infrasons et les effets de lumière (de jour: les alternances ombre - lumière et de nuit les flashs de signalisation). Cela a une influence sur l'impression de confort et éventuellement sur la santé (Altmoos, 2014a). On va ainsi décrire les pathologies en lien avec le bruit et les infrasons ${ }^{4}$. En ce qui concerne les conséquences exactes sur les êtres vivants et les hommes il y a encore beaucoup d'incertitudes et un déficit de recherches : les études réalisées par les firmes éoliennes tendent à dédramatiser le problème, alors que d'autres études préconisent une implantation des éoliennes à des distances d'au moins 5 à $10 \mathrm{~km}$ des habitations à cause des nuisances dues aux infrasons et aux effets d'ombre et de lumière ${ }^{5}$. En tout cas, l'espace proche des parcs éoliens est dévalorisé pour le logement et les activités de loisirs et doit être utilisé autrement; ce qui a des conséquences en matière de planification.

Les éoliennes sont aussi sources de risques et d'accidents, même si bien sûr ils ne sont pas aussi dramatiques que ceux dus aux installations nucléaires. Les éoliennes peuvent provoquer des projections de glace, subir des incendies, ou perdre certains éléments, ou encore être à l'origine d'une recrudescence des éclairs dans leur environnement. En principe, l'industrie éolienne effectue des tests de résistance du matériel et prend de nombreuses mesures de précaution pour éviter les accidents ${ }^{6}$. Malgré cela, il y a quand même de nombreux accidents à la suite d'éclairs, d'incendies, de projections de glace 
ou de chutes d'éléments d'éoliennes consécutifs à des erreurs de montage ou d'usure du matériel; même si jusqu'à présent peu de gens ont été sérieusement blessés.

Il n'existe pas d'assurance, ni de preuves en ce qui concerne l'importance des dangers pour la santé. Avec la poursuite de la construction d'éoliennes dans les paysages on est en train de faire une expérience y compris sur les hommes. En principe, ce sont plutôt les personnes sensibles qui devraient être affectées. Le fait qu'il s'agisse d'effets d'autosuggestion reste une question ouverte ${ }^{7}$. En tout cas le sentiment que le „Heimat“ et le paysage sont dégradés peut avoir des effets négatifs sur l'identité et le confort moral (Altmoos 2014a, b).

21 La chance est de minimiser les conséquences négatives pour les gens grâce à des distances de sécurité par rapport aux zones habitées. Les distances actuelles sont cependant souvent trop petites et insuffisantes. Il n'y a qu'en Bavière que les distances ont été portées récemment à au moins $2 \mathrm{~km}$ avec des procédures d'autorisation en conséquence. Mais de plus petites distances sont aussi imposées politiquement (en Rhénanie-Palatinat théoriquement $800 \mathrm{~m}$, en réalité souvent moins) car sinon dans un pays aussi densément peuplé que l'Allemagne il ne serait pas possible de construire de nouveaux sites éoliens.

Cependant, on a tendance à construire les grands parcs éoliens loin des lieux habités. Cela ne dérange pas directement les gens, notamment dans les agglomérations; mais cela concerne un autre problème : la biodiversité.

\section{B. Les effets sur la biodiversité: animaux, biotopes et écologie du paysage}

Les effets du développement de l'éolien sur les animaux et les biotopes font l'objet de différentes recherches. Même si ces recherches sont encore relativement récentes et lacunaires, on peut déjà faire des analyses préliminaires et des évaluations (synthétisées par Altmoos 2014a): le risque de collisions avec les oiseaux est bien connu, en particulier pour les grands oiseaux en fonction de l'emplacement, des espèces et de la saison (par exemple pour le milan royal, Milvus milvus, Eichhorn et al. 2012). La mortalité peut en effet être probablement réduite par un choix judicieux de l'emplacement. Mais avec des espèces rares, même de faibles taux de mortalité peuvent être graves.

Depuis peu, les chauves-souris et leur vulnérabilité aux éoliennes sont au centre des préoccupations : soit elles meurent immédiatement (voir chiffres ci-dessous), soit plus tard dans leurs refuges après des blessures ou suite à des séquelles. Cela se fait soit par collision avec les rotors, soit par un "barotraumatisme ', une lésion des poumons consécutive à des différences de pression de l'air due à la rotation du rotor sur la trajectoire de vol (Baerwald et al., 2008). Dans d'autres études, l'importance des barotraumatismes est cependant critiquée ${ }^{8}$. Mais quelle que soit la cause des décès, selon les études scientifiques systématiques effectuées sur des échantillons, leur extrapolation conduit à évaluer à 250000 en Allemagne et à 600000 aux États-Unis le nombre de chauves-souris directement victimes des éoliennes (Allemagne: Uni de $2013^{9}$ Etats-Unis: Hayes 2013). En outre, cela dépend aussi des mouvements des insectes que les éoliennes suivent (Rydell et al. 2010). Il y a aussi certainement de fortes mortalités d'insectes sur les éoliennes, bien que leur importance n'est pas bien connue. 

ne sont même pas respectés. opportunistes ou de charognards. sur d'autres espèces et des habitats entiers. 2002).

En arrêtant les rotors dans les principaux temps d'activité, le risque pour les chauvessouris et les oiseaux pourrait être réduit; mais on ne sait pas encore si cela suffit et si c'est vraiment utile. Il reste aussi le problème des perturbations des habitats et du paysage par les éoliennes elles-mêmes. Ces problèmes vont au delà du thème de la conservation des espèces et posent des questions concernant l'écologie des paysages.

Les problèmes que posent les éoliennes sont particulièrement importants dans les forêts. Les zones défrichées et en particulier la fragmentation horizontale et verticale de la forêt par les parcs éoliens et leurs voies d'accès sont au total déjà énormes dans les massifs de la Rhénanie-Palatinat. À l'inverse, on peut voir de manière positive l'apparition de nouvelles clairières et de micro-habitats ouverts comme ce pourrait aussi être le cas dans la dynamique des forêts naturelles; mais cela ne pèse généralement pas beaucoup par rapport aux importantes perturbations de l'habitat et à la fragmentation. Bien que l'Agence fédérale allemande pour la conservation de la nature (BfN 2011) ait élaboré sur une base scientifique des recommandations pour la prévention des conflits avec les éoliennes dans la forêt, dans la réalité ces compromis

7 La construction d'éoliennes dans les forêts progresse. Tout aussi grave est l'implantation d'éoliennes sur les lisières forestières, ou au milieu des écosystèmes de prairies. Ce sont souvent des centres de biodiversité, ou alors y vivent des animaux sensibles aux perturbations des éoliennes qui disparaissent au profit d'espèces

Les ressources abiotiques sont aussi atteintes dans la mesure où les fondations profondes pour les grandes éoliennes et leurs voies d'accès peuvent perturber le régime hydrologique (y compris l'approvisionnement en eau) et ainsi avoir des effets négatifs

Avant chaque permis de construire pour une éolienne il existe en Allemagne une procédure de contrôle qui concerne aussi l'ensemble de la biodiversité. Dans la pratique cependant ces études sont souvent insuffisantes du point de vue méthodologique, ou alors orientées en faveur de l'industrie éolienne. Les organismes publics qui les contrôlent et donnent les autorisations sont soit surchargés, soit sous une forte pression politique afin de favoriser le développement de l'éolien. Aussi voit-on toujours des sites particulièrement sensibles être construits (Altmoos $2014 \mathrm{a}, \mathrm{b}$ ). La biodiversité dans son ensemble est menacée et même les espaces protégés ne sont pas tabous, à l'exception de quelques petits espaces de protection de la nature.

\section{Changements paysagers, consommation d'espace et impact de la production décentralisée}

Au total, le plus important et le plus visible est l'impact paysager qui est bien plus qu'une question esthétique et doit être considéré comme un indicateur de multiples nuisances pour les hommes et la biodiversité. La question esthétique est néanmoins importante car elle contribue à l'identité et au bien-être des êtres humains (Wöbse

Une des conséquences problématiques est qu'avec l'importance de la construction d'éoliennes il n'y a pratiquement plus d'horizons dégagés. Un nouveau type de paysage apparaît: un paysage énergétique modelé par l'industrie, même dans des espaces 
ruraux périphériques qui jusqu'à présent avaient été peu touchés par le secteur énergétique (voir l'exemple du Bade-Wurtemberg (Nägele, 2012) ou celui du sud-ouest de l'Allemagne (Altmoos, 2014b)). Les impacts paysagers des grandes éoliennes sont majoritairement jugés comme étant négatifs, même si elles exercent une certaine fascination et l'intérêt d'une minorité (note 1).

Il faut bien reconnaître que les paysages ont toujours changé (voir Küster, 1995) et que naturellement ils peuvent continuer à changer et même ils le doivent pour différentes raisons (par exemple Schaich \& Konold 2011). Mais le développement actuel de l'éolien, dans ses dimensions et son impact spatial, est un phénomène nouveau et va bien audelà des échelles de changement observées jusqu'à présent:

Il est particulièrement grand: la hauteur des nouvelles installations atteint celle des gratte-ciel des grandes villes (plus de $150 \mathrm{~m}$ et même plus de $200 \mathrm{~m}$ ) et elles sont regroupées dans de vastes parcs (plus de $100 \mathrm{ha)}$ traversant l'espace autrefois libre, bien au-delà des dimensions humaines habituelles (voir Wöbse 2002).

34 (b) Il est brutal, parce que le développement se fait rapidement, par-dessus la tête de la plupart des gens. Relativement peu de gens décident de faire quelque chose qui devient soudain dominant pour tous.

(c) Il a de grands effets spatiaux, notamment quand les espaces entre les parcs sont traditionnellement faibles comme c'est le cas en Allemagne du sud-ouest. Presque tout l'espace du Land est marqué à une dimension inconnue jusqu'alors.

36 (d) Il a un effet de nivellement, car avec la construction en hauteur, des paysages qui étaient auparavant différents ont la même empreinte industrielle. C'est un inconvénient pour la différenciation des paysages sur laquelle repose par exemple le tourisme, mais aussi les identités régionales.

37 Au total, il s'agit de la transformation spatiale d'origine anthropique la plus visible et la plus profonde qui se soit produite dans le paysage des civilisations au cours des temps historiques. Ce superlatif se justifie d'autant plus qu'à cela s'ajoutent les monocultures de plantes énergétiques et la transformation des cours d'eau pour l'hydroélectricité dans le cadre du «tournant énergétique», avec des effets plutôt horizontaux que verticaux.

Un des avantages souvent avancés des éoliennes est leur faible emprise au sol. On a ainsi calculé qu'on aurait besoin de n'utiliser que de 1 à $3 \%$ de la surface du pays pour implanter les dizaines de milliers d'éoliennes nécessaires pour assurer l'approvisionnement complet en combinaison avec d'autres énergies renouvelables. La Rhénanie-Palatinat, la Hesse et le Bade-Wurtemberg ont annoncé leur objectif d'utiliser au maximum $2 \%$ de leur superficie pour les installations éoliennes. En ce sens, l'éolien apparaît comme une forme d'énergie particulièrement efficiente.

Cela est renforcé par le „Repowering“: en raison de l'usure du matériel, les éoliennes ont actuellement en moyenne une durée de vie maximale de vingt ans. La plupart du temps elles sont remplacées avant même ce délai par des installations plus productives. Celles-ci sont cependant plus grandes et ont un impact plus important sur les paysages, les espaces de vie, les hommes et les animaux. Par contre, on a ainsi besoin de moins de surface pour la même production d'énergie.

40 Un autre grand avantage est que les éoliennes peuvent toujours être démontées et recyclées. Le développement pourrait alors rapidement être inversé ou modifié. Cela fait une grande différence avec beaucoup des autres installations industrielles ou 
énergétiques. Cependant, l'expérience vécue jusqu'à présent montre qu'une fois occupé par une éolienne, un site est plutôt plusieurs fois „repowerisé“ qu'abandonné. Et les fondations profondes des grandes éoliennes sont pratiquement indémontables.

Cependant, la faible proportion de surfaces directement bâties est trompeuse: il s'agit surtout de la qualité des surfaces affectées, par exemple si des biens protégés sont concernés. Et du fait de la hauteur et de la puissance des grandes éoliennes, les effets sont très étendus malgré la faible importance des surfaces directement bâties. De plus, à l'intérieur des parcs éoliens, il faut conserver de grandes distances entre les différentes installations afin qu'elles ne diminuent pas la production éolienne. La surface des parcs éoliens en augmente d'autant plus. En fonction de la disposition spatiale des éoliennes, on peut estimer que pour $2 \%$ de surface construite ce sont en fait 80 à $90 \%$ de l'ensemble du paysage qui sont affectés. Et d'ores et déjà en RhénaniePalatinat, il n'existe plus aujourd'hui d'espace de 20 à $30 \mathrm{~km}$ d'un seul tenant sans éolienne visible.

L'effet de domination est d'autant plus accentué que l'on choisit de préférence des sites venteux qui par nature même sont situés sur les collines les plus élevées et dans des forêts particulièrement bien exposées, le plus souvent à l'écart des zones habitées. Construire sur ces sites est d'une part économiquement plus rentable; mais d'autre part cela a de plus grandes conséquences et un effet destructeur sur les espaces naturels, même si les zones habitées sont ainsi épargnées. Cependant, avec la loi EEG sur les énergies renouvelables, on subventionne aussi des sites relativement peu venteux dans des zones basses; si bien que la consommation de surface par l'énergie éolienne est encore renforcée.

Entre temps, il est même prévu d'occuper les derniers grands espaces libres d'éoliennes $\mathrm{du}$ sud-ouest de l'Allemagne. Par exemple, dans la réserve de biosphère francoallemande du Pfälzerwald et des Vosges qui est pour le moment dépourvue d'éoliennes, il existe du côté allemand plus de 40 projets de grandes éoliennes. Dans le parc naturel de Saar-Hunsrück qui compte déjà de nombreuses éoliennes, au moins 80 éoliennes supplémentaires doivent être installées. Enfin, dans le parc naturel du Soonwald-Nahe, un minimum de 30 éoliennes supplémentaires doivent être construites alors que les environs immédiats de ce parc sont déjà saturés d'installations. On peut certes penser que tous ces projets ne verront pas le jour. Néanmoins, il suffit qu'une petite partie d'entre eux soient réalisés pour que, dispersés dans ces espaces, on arrive à une couverture presque complète d'installations éoliennes (figure 2). 
Figure 2. Représentation cartographique simplifiée du mitage des espaces par les éoliennes à partir de la région autour du parc naturel de Soonwald-Nahe (cadre vert), un des espaces encore relativement épargné par les éoliennes en Rhénanie-Palatinat (cartographie et recherches de Michael Altmoos, janvier 2014).

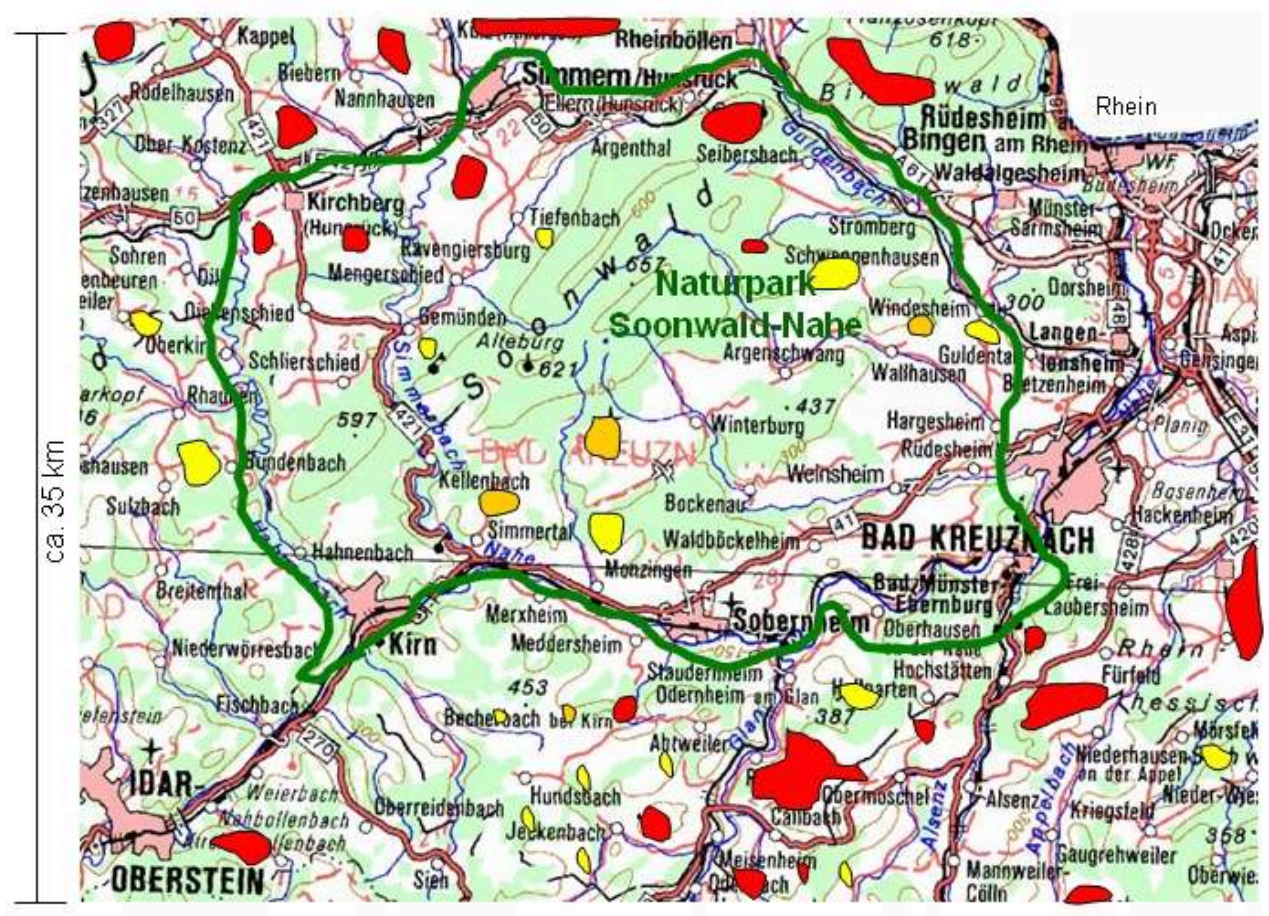
ca. $50 \mathrm{~km}$

2a: accroissement dans l'espace: rouge: parcs éoliens existants. II subsiste de grandes surfaces dans le parc naturel qui ne sont pas encore mitées par les éoliennes, mais où de nouvelles installations sont prévues: Orange: parcs éoliens en voie d'autorisation. Jaune : parcs prévus (représentation simplifiée des surfaces et suivant les limites externes des parcs éoliens). 


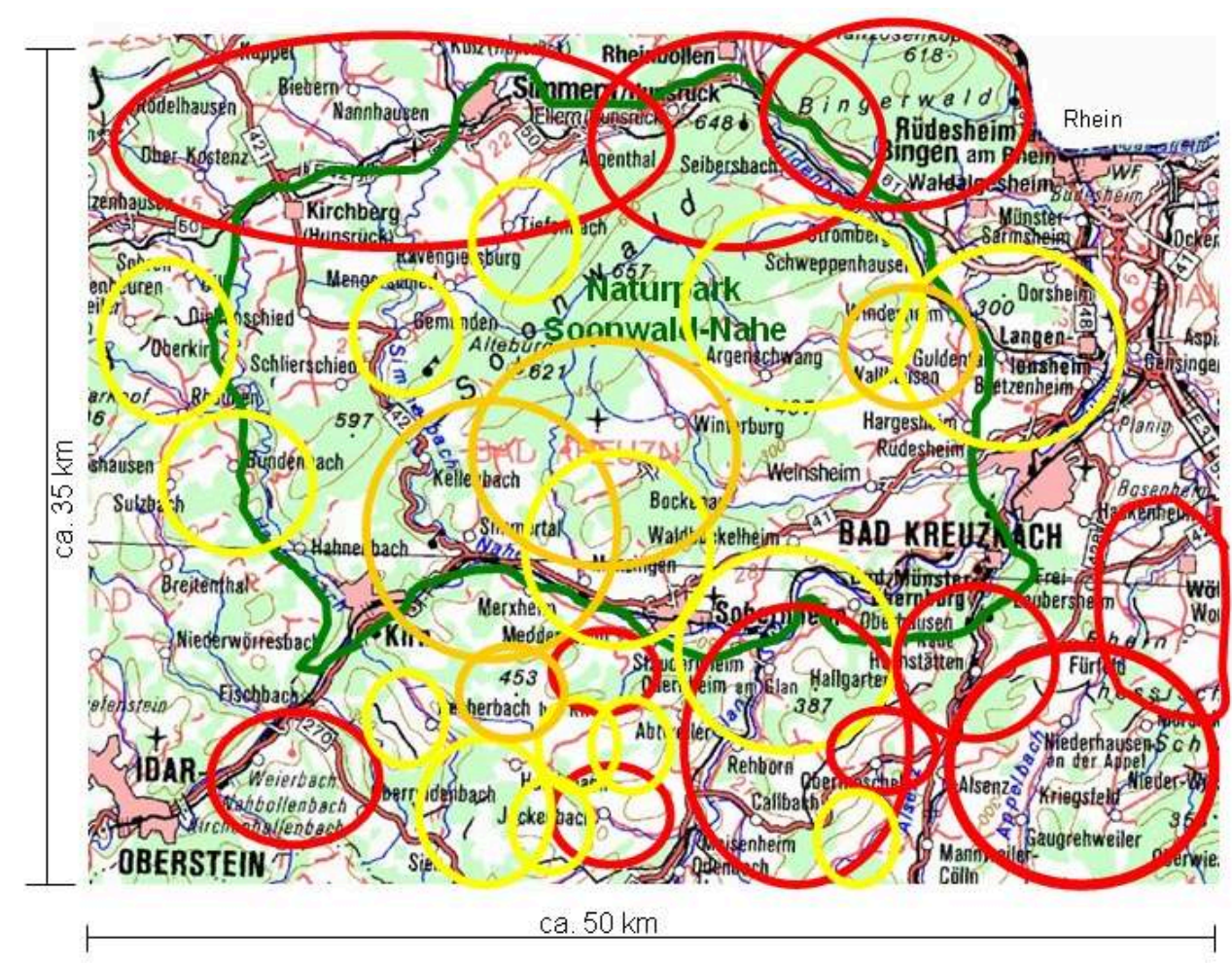

2b: estimation de l'effet visuel: inégalement important en fonction de la hauteur et de la surface des parcs éoliens, ainsi qu'en fonction de leur exposition (3 à 8 km sur des zones circulaires représentées de manière simplifiée). En réalité, des variations de cet effet visuel suivant les vallées et les hauteurs. La proximité ou les effets visuels de domination (à l'intérieur des cercles) peuvent être considérés comme un indicateur de divers problèmes qui sont incontestables.

Une caractéristique fondamentale du développement des énergies renouvelables et en particulier de l'éolien est qu'à la place de la centralité des grandes installations de production elles se déploient de manière décentralisée (voir Deshaies 2013, Smil 2010). Le caractère décentralisé est souvent considéré comme un avantage car il est associé à de nouvelles valeurs ajoutées locales, par exemple sous la forme de nouvelles sources de revenus dans des espaces ruraux autrefois défavorisés. Cela peut être aussi perçu comme un désavantage dans la mesure où, au lieu d'une valeur ajoutée locale il y a en fait destruction, mitage et impact démesuré par la technique énergétique, précisément dans des espaces ruraux et naturels qui étaient „intacts“.

Un impact négatif sur le tourisme est à craindre, notamment sur le type de tourisme lié aux „beaux“ paysages, même si cela n'est pas encore bien établi (il existe plusieurs études non publiées avec des résultats différents, par exemple Coch \& Riesterer 2013).

Dans tous les cas cependant le développement de l'éolien et le caractère décentralisé des énergies renouvelables signifie un changement structurel profond : d'un réseau d'axes centralisés pour la production et la distribution d'énergie à un réseau décentralisé et couvrant presque complètement les surfaces (figure 3).

Cela est lié étroitement à la question du réseau: des lignes électriques nouvelles, des réseaux de distribution décentralisés sont nécessaires. L'adaptation du réseau est nettement en retard sur le développement de l'éolien. Sans un réseau d'infrastructures bien pensé il ne peut pourtant pas y avoir de „tournant énergétique“ (Bachem \& Buchal 2013). Si le réseau est restructuré en conséquence alors cela entraine une 
fragmentation supplémentaire des paysages et des nuisances pour les hommes et les milieux naturels. Un véritable cercle vicieux!

\section{L'acceptance, „les lignes de fracture et les nouveaux conflits“}

En ce qui concerne les changements paysagers, la recherche en sociologie de l'environnement a mis en évidence les effets de l'habitude. En fait, les gens se sont toujours étonnamment adaptés à de nouvelles conditions et l'ont fait de manière fascinante. Alors que l'ancienne image du paysage ou de l'identité propre disparait, de nouveaux rapports de production sont parfois assez rapidement acceptés comme normaux („Shifting Baseline Syndrom“, voir un résumé par exemple dans Rost 2014).

On peut déjà s'en rendre compte dans l'est du Hunsrück (Rhénanie-Palatinat) qui est fortement marqué par les éoliennes: quelques personnes interrogées sur le paysage récemment transformé par l'éolien considèrent que c'est normal; beaucoup de personnes sont même indifférentes. Les enfants représentent de grandes éoliennes dans leurs dessins de paysages. Et beaucoup d'adultes considèrent les éoliennes comme bénéfiques car elles sont à la fois sources d'optimisme financier et industriel. Pourtant, autrefois, un horizon dégagé et des forêts continues allaient de soi dans cet espace rural au paysage attractif. C'était même un emblème positif pour le tourisme. Aussi, beaucoup d'autres gens ressentent-ils cette mutation comme étant négative, notamment les habitants qui, quel que soit leur âge, apprécient les milieux naturels et la nature et constatent leur disparition. Le sentiment de la perte du „Heimat" est aussi apparent. En conséquence, la résistance à ces évolutions augmente (note 2). La région se divise. Les lignes de fracture ne sont pas indéfiniment extensibles, même si d'après Rost (2014) les gens peuvent réagir avec une certaine souplesse. La recherche sociologique sur ces réactions en est encore à ses débuts.

Lors des conflits sur la poursuite du développement des énergies renouvelables, les lignes de partage entre les "défenseurs de l'environnement“ et les „économistes“ s'effacent. Les intérêts entre les espaces périphériques et urbains sont remis en cause (Gailing \& Leibenath 2013). La transition énergétique fait en sorte que les différentes catégories d'acteurs et de situations s'interpénètrent. Cela peut être interprété négativement en raison des mécontentements qu'elle génère, mais aussi positivement par le rafraichissant changement qu'elle apporte (Ackermann et al. 2001). Ainsi en Allemagne, même les sociétés de protection de la nature se disputent entre elles, alors qu'elles étaient autrefois d'accord pour plus de nature et de protection du climat.

D'après nos observations, les partisans de l'énergie éolienne, à l'orientation plus technicienne, donnent la priorité à la protection du climat et se démarquent ainsi de ceux qui refusent trop d'éolien et considèrent que les paysages, la biodiversité et la protection $\mathrm{du}$ climat forment un tout que menace l'excès d'éoliennes. Il y a actuellement un problème d'incompréhension entre les différentes positions. À cela s'ajoutent les intérêts financiers directs des profiteurs de l'éolien. Tout cela est cependant dominé par le fait que beaucoup de gens sont indifférents car ils ont d'autres soucis, ou alors ils n'ont aucune relation avec la nature et le paysage. En définitive tout le monde se retrouve avec étonnement devant le plus grand changement paysager que l'on ait vu, accompagné d'une somme importante de problèmes. 


\section{Les limites de la transition énergétique vers les énergies renouvelables}

\section{A. Les contributions à l'approvisionnement énergétique renouvelable}

$\mathrm{Au}$ vue des problèmes présentés pour les hommes et la biodiversité la question se pose de savoir quel gain énergétique cela représente-t-il. On peut d'abord constater que si les éoliennes se sont autant développées, c'est à la suite de l'amélioration de leur productivité. Les partisans de l'éolien parlent de la méthode de production d'énergie sans émissions de gaz, de la meilleure que l'on puisse avoir sur terre. Beaucoup d'emplois en dépendent, environ 3000 selon le gouvernement de Rhénanie-Palatinat (en janvier 2014). Et justement, sur les sites venteux, en théorie, la production peut être importante. C'est une grande chance. Mais en pratique il y a aussi des limites.

La véritable productivité de l'éolien est de plus en plus remise en cause. Malgré un haut niveau de développement, l'énergie éolienne ne fournissait en 2012 que 7,3\% de la production électrique en Allemagne (Bachem \& Buchal 2013). Au cours de l'année 2014, la part des énergies renouvelables atteignait certes presque $25 \%$ du mix énergétique (données du syndicat de l'électricité, BDEW); mais l'énergie éolienne représentait une part en croissance de seulement $10 \%$ de la production totale. C'est beaucoup si l'on songe qu'il y a 10 ans l'électricité éolienne était à peine mesurable; mais c'est très peu au regard de la somme des installations déjà réalisées et des problèmes posés à l'homme, à la nature et au paysage.

Il faut aussi tenir compte du fait que durant les périodes venteuses, il y a déjà plus de courant éolien que nécessaire, alors que la plupart du temps la contribution de l'énergie éolienne à la production de courant est faible. Aussi doit-on même souvent acheter en France de l'électricité nucléaire, précisément la forme d'énergie que le tournant énergétique allemand est censé éviter. C'est pourquoi, dans cet article, nous avons renoncé aux chiffres souvent impressionnants de capacités théoriques éoliennes installées en MW (une bonne vue d'ensemble dans Deshaies, 2013). Ce qui est important c'est la production effective d'électricité qui est finalement assez faible. Pour augmenter fortement la part de l'éolien, il faudrait construire des dizaines de milliers de nouvelles installations (avec encore bien plus de transformations paysagères et de problèmes que ceux existants).

\section{B. La question clef du stockage de l'énergie}

L'énergie éolienne est comme l'énergie solaire une forme d'énergie volatile (elle est naturellement fluctuante). Quand le vent souffle fort, il existe déjà aujourd'hui dans beaucoup de régions du sud-ouest de l'Allemagne, un excédent de courant électrique qui est envoyé gratuitement vers d'autres régions d'Allemagne, ou en France. Cela peut être vu comme un avantage ou un succès, surtout pour la France: mais cela souligne aussi les désavantages de cette forme d'énergie: les variations de la production sont un défi pour la technique des réseaux et la stabilité de l'approvisionnement électrique (Bachem \& Buchal 2013). Il faut nécessairement des solutions de stockage si l'on veut que l'électricité éolienne soit disponible plus longtemps et en cas de besoin. Le stockage de l'énergie est un problème central et la question clef du „tournant énergétique“. 
Pour le moment, force est de constater qu'il n'y a pas encore de possibilités de stockage adaptées, malgré des recherches intensives sur ce sujet et bien qu'il y ait déjà 40 centrales hydroélectriques de pompage-turbinage en Allemagne, une possibilité de stockage éprouvée et peut-être la seule technique mûre de stockage en grand (Meiller et al. 2011). Les partisans de l'éolien voient le développement futur de la recherche avec optimisme; les opposants rétorquent qu'il existera des solutions de stockage flexibles au plus tôt dans deux décennies.

57 Afin d'augmenter significativement la part de l'éolien comme cela est politiquement souhaité, il serait nécessaire de construire dès maintenant de nouveaux grands réservoirs (des centrales de pompage-turbinage). Quelques-uns sont planifiés. Mais pour cela, de nouvelles dégradations de la nature et du paysage sont nécessaires dans la mesure où des collines et des montagnes entières avec leurs cours d'eau seront transformées.

\section{La multiplication des effets négatifs}

Le problème du stockage fait que les centrales thermiques ou nucléaires doivent continuer à marcher en réserve, même si c'est de façon réduite, par moment. Il faut alors entretenir un double parc de centrales de production avec les désavantages écologiques et économiques qui en résultent.

59 À cela s'ajoutent les doutes sur la capacité de l'énergie éolienne à abaisser les émissions de dioxyde de carbone. On peut voir de manière positive le fait que le fonctionnement des éoliennes n'émette pas de gaz et peu de matières toxiques, sauf pour leur fabrication et les matières premières qu'elles nécessitent (ou lors d'accidents). Lorsque pour construire des éoliennes l'on défriche de la forêt qui sert de réservoir à dioxyde de carbone et qui peut aussi contenir du vieux bois et des espèces rares, le bilan écologique peut alors être négatif au lieu d'être positif en fonction du modèle de calcul utilisé.

60 Le mécanisme actuel du commerce international des émissions fait que l'on émet ailleurs plus de dioxyde de carbone lorsqu'au mieux on parvient à les réduire par les éoliennes. Afin d'empêcher les ruptures d'approvisionnement et les problèmes de stockage on construit même de nouvelles centrales thermiques à charbon, alors que des centrales à gaz efficaces et déja existantes deviennent non rentables (une situation complexe). Un concept d'ensemble n'est pas encore identifiable.

61 Dans ce contexte, ce n'est donc pas malgré, mais précisément à cause du fort développement des énergies renouvelables que les émissions de dioxyde de carbone de l'Allemagne ont augmenté au cours des dernières années (et n'ont pas baissé). À cela s'ajoute le fait que les matières premières utilisées (en partie les terres rares) dans les pays d'origine peut être problématique. Au total, force est de constater qu'au moins pour le moment, l'énergie éolienne ne sert pas à la protection du climat et qu'elle n'a pas l'effet escompté bénéfique pour l'environnement. En résumé, avec les problèmes évoqués précédemment il se produit une multiplication des dommages environnementaux:

62 (a) directs par les éoliennes elles-mêmes (les effets sur les hommes, les animaux et le paysage, voir ci-dessus), 
63 (b) indirects avec la construction des infrastructures nécessaires, en l'occurrence les chemins d'accès et les nouvelles lignes électriques à la suite du système décentralisé. La fragmentation des milieux naturels est au total importante, tournant énergétique avec les problèmes qui lui sont liés (ceux du passé et à venir): en 3a on a symbolisé l'état initial avec beaucoup d'espaces naturels (en vert) et les infrastructures énergétiques en rouge. En $3 b$ on a représenté le paysage qui jusqu'à récemment était dominé par des centrales thermiques ou nucléaires avec les lignes électriques correspondantes. Quelques espaces naturels en sont déjà victimes ou sont menacés. La vignette $3 \mathrm{c}$ illustre le nouveau développement anarchique qui se produit actuellement en Allemagne: des installations énergétiques décentralisées qui certes ont toujours existé en petit nombre et de petite taille, mais qui se sont multipliées et atteignent des dimensions gigantesques (ici l'énergie éolienne). Il manque une coordination d'ensemble. La conséquence est une fragmentation du paysage et une destruction des espaces naturels qui n'avaient pas encore été atteints. Comme il manque des installations de stockage, le parc conventionnel de centrales doit aussi en grande partie continuer à fonctionner. Contrairement à autrefois, les énergies dominent presque tout l'espace.

68 Comment cela peut-il continuer dans l'avenir, c'est bien là la question. Il y a deux scénarios: la figure $3 \mathrm{~d}$ montre la poursuite de l'évolution actuelle avec l'idée souvent présentée en Allemagne que l'on pourra renoncer complètement au complexe des énergies fossiles et nucléaires par encore plus de développement des énergies renouvelables. En conséquence, il faut encore beaucoup plus de grandes éoliennes et aussi beaucoup plus de réservoirs de stockage destructeurs de paysage. En conséquence, le paysage est de plus en plus fortement fragmenté, même si dans le meilleur des cas de petites erreurs peuvent être corrigées dans la mesure où, après 
quelque temps on démonte progressivement des éoliennes mal situées pour les installer ailleurs. L'image d'ensemble ne change pratiquement pas.

Figure 3. Représentation simplifiée et schématique de l'évolution des espaces sous l'influence des infrastructures énergétiques (infrastructures énergétiques en rouge, zones d'habitat en gris, voies de communication en lignes noires. Explications dans le texte).

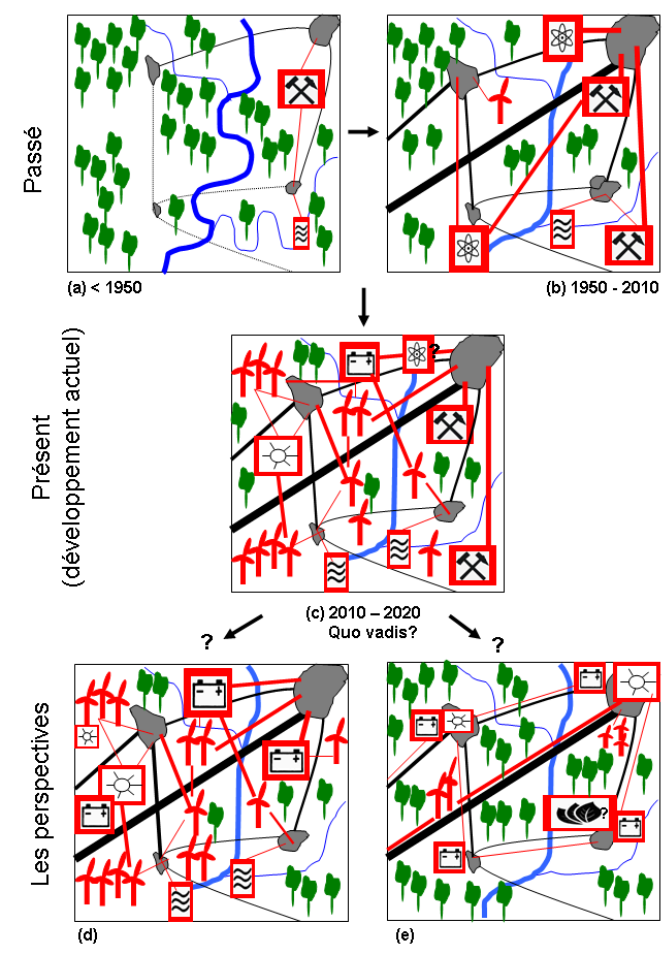


Figure 4. Schéma des problèmes et solutions pour intégrer l'éolien dans une transition énergétique environnementalement supportable (explications dans le texte).

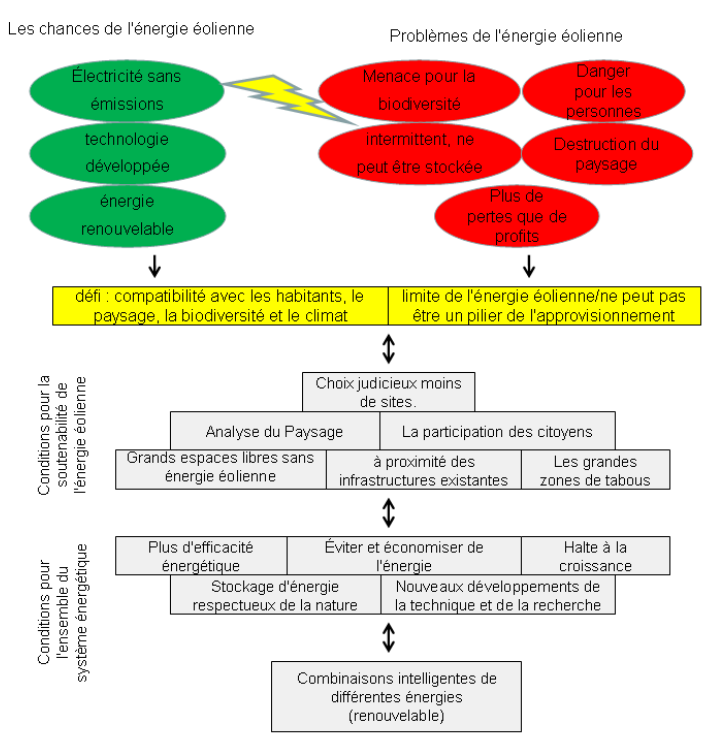

\section{Discussion, conséquences et alternatives: du „conflit autour des paysages" à la "réconciliation avec l'énergie“"}

Pour résoudre les problèmes présentés ci-dessus et améliorer la situation, il faut considérer les questions énergétiques et la protection du climat comme un ensemble et ne pas les réduire aux questions techniques de la production de courant et de diminution des émissions. Dans une vision écologique, il est visible que presque toutes les mesures de protection de la nature et de cadre de vie servent en même temps à la protection du climat: la conservation, ou la reconstitution de forêts continues proches de l'état naturel (auquel appartiennent les animaux comme les chauves-souris), ainsi que d'autres types d'espaces naturels (qui peuvent aussi servir à stocker l'eau et à réguler le climat) sont des exemples connus. À l'inverse, les mesures de protection du climat trop exclusivement techniques et notamment les éoliennes comme il a été montré ci-dessus, dégradent les espaces naturels et finalement ont un effet négatif sur le climat; ce qui n'est pas pris en considération dans les visions purement techniciennes.

En conséquence, il faudrait développer une planification intégrative d'ensemble qui mette en relation les principales questions techniques (pour une présentation d'ensemble : Bachem \& Buchal 2013) avec les questions de protection de la nature et les considérations géographiques. Car elles sont par définition destinées à gérer et à résoudre les conflits spatiaux; c'est la raison d'être d'une bonne planification des paysages (Jessel 1998). 
71 Dans ce sens, à la suite des problèmes évoqués ci-dessus, on peut dresser sept lignes directrices pour permettre concrètement d'utiliser durablement l'énergie éolienne de telle sorte que l'on puisse utiliser ses avantages en limitant ses inconvénients (figure 4):

72 L'énergie éolienne doit être fortement limitée car ses inconvénients s'amplifient. Cela signifie que l'on peut utiliser l'éolien, mais pas à grande échelle. Notamment dans le sud-ouest de l'Allemagne le niveau de saturation est déja presque partout atteint; il ne reste que quelques grands espaces sans éoliennes qui devraient être absolument préservés. Pour les autres Länder dans lesquels l'éolien est pour le moment peu présent il existe les possibilités suivantes:

73 Disposer quelques éoliennes de manière intelligente et avec de grands écarts entre elles (avec une coordination à l'échelle du Land et pas au niveau de petites unités territoriales). Comme on l'a vu, une planification à l'échelle locale n'est pas adaptée au $\mathrm{vu}$ des dimensions des installations et des contraintes d'ensemble du tournant énergétique. Il faut de grandes distances minimales par rapport aux habitations (par précaution vis-à-vis de la santé et des risques, plus de $2 \mathrm{~km}$ ), ainsi que pour certaines zones naturelles et pour les zones d'habitat des espèces protégées, même quand ces espaces sont des sites venteux (distances à respecter pour le milan royal Milvus milvus de Eichhorn et al. 2010). En relation avec cela :

74 Préserver de très grands espaces „tabous“ sans éoliennes, car sinon il risque d'y avoir saturation de l'espace. Il devrait s'agir tout d'abord des parcs naturels dans leur ensemble (en France les parcs régionaux), des réserves de biosphère, des paysages protégés, des espaces naturels protégés, des zones Natura 2000 et des parcs nationaux, ainsi que de leur environnement, mais aussi des quelques espaces encore vierges de grandes installations, même s'ils n'ont pas jusqu'à présent de statut de protection.

Ce qui devrait aussi prévaloir: prendre le paysage au sérieux parce qu'il a une valeur pluridimensionnelle que l'on considère faussement comme étant une affaire de goût. Pour cela, il faut utiliser ou développer des études paysagères fondées sur des données géographiques et des évaluations de l'image des paysages (par exemple Roser 2013 pour Baden-Wurtemberg). À partir de cela :

76 Privilégier pour l'installation des éoliennes des espaces qui sont déjà fortement affectés par les infrastructures. Il convient alors de placer les éoliennes à proximité des autoroutes ou des grandes zones d'activités, parce que cela réduit la consommation de nature et que c'est souvent plus économique. Cela économise les coûts d'accès au réseau et réduit la fragmentation de l'espace par de nouvelles lignes électriques. Il faut aussi conserver suffisamment de distance par rapport aux zones d'habitat.

77 Faire participer les habitants bien au-delà de la commune dans laquelle se trouvent les installations (parce que nous sommes dans des sociétés démocratiques et qu'un tel changement paysager qui concerne tout le monde, ne devrait pas être réalisé brutalement et à l'initiative de quelques acteurs).

78 Moins de gigantomanie pour les installations éoliennes, aussi bien en hauteur que pour les surfaces couvertes parce que cela affecte particulièrement le paysage et les hommes. De petites installations peuvent aussi apporter une contribution, mais elles ne doivent pas pour autant consommer plus d'espace que les grandes éoliennes. Il faut aussi un usage raisonné de l'efficacité: les sites venteux doivent certes être privilégiés 
par rapport à ceux qui le sont moins. Mais si de tels sites (par exemple des hauteurs boisées) sont particulièrement sensibles en ce qui concerne le paysage et la biodiversité, ils devraient alors suivant les critères 2 à 6 , être préservés au profit de sites moins venteux. L'efficacité est importante, mais ne doit pas être la mesure de toute chose.

Cela limite fortement le potentiel de l'énergie éolienne. Mais c'est la seule possibilité de rendre l'énergie éolienne environnementalement supportable dans le cadre du „tournant énergétique“ (et pas seulement considéré comme un pilier central comme aujourd'hui dans le sud-ouest de l'Allemagne).

La figure 3e dessine une vision spatiale alternative de ce que pourrait être le tournant énergétique en intégrant l'énergie éolienne de manière avantageuse (en réconciliant les exigences écologiques, économiques et sociales d'un véritable tournant énergétique):

81 Différentes énergies renouvelables peuvent et devraient être combinées intelligemment entre elles, de telle sorte qu'au contraire de ce qui se produit actuellement en Allemagne du sud-ouest, la nature et le paysage soient pris au sérieux comme critères de référence et soient peu construits ou dégradés (figure 4). Cela est valable aussi pour d'éventuels nouveaux développements dans le secteur énergétique (symbolisés par le figuré avec des feuilles de la figure 3e). Il s'agit par exemple: de l'énergie solaire sur les toitures (modernes) dans les zones d'habitation et dans les zones d'activités existantes, au lieu de les implanter dans les champs. L'utilisation des déchets de la biomasse et une consommation de bois raisonnée, autrement dit „durable“, de telle sorte que l'on puisse avoir plus de zones de nature „sauvage“. Des installations hydrauliques sur des secteurs déjà équipés et construire ou préserver des escaliers à poisson (ainsi que d'autres combinaisons douces de différentes formes d'énergie afin que si chaque forme d'énergie peut avoir des effets négatifs, une solution est néanmoins possible (pour les détails, voir la note 10 concernant la préservation de la nature $)^{10}$.

Toutes les sources d'énergie renouvelable et pas seulement l'énergie éolienne devraient être à proximité des infrastructures existantes ou à proximité relative du consommateur; si bien que de grands espaces demeurent sans infrastructures énergétiques. Ainsi, le réseau énergétique futur serait plus décentralisé qu'aujourd'hui, mais pas de manière aussi serrée, chaotique et fragmentée que dans la phase actuelle dans le sud-ouest de l'Allemagne (figure $3 c+d$ ).

La vision qui consiste à sortir du complexe des énergies fossiles et nucléaires et de ses inconvénients est importante. De nouveaux développements présentent une dimension encore inconnue (une éventuelle fusion nucléaire dépourvue de risques? Un recyclage des déchets nucléaires?). De nouveaux réservoirs de stockage flexibles et moins dommageables pour les paysages (smarts grids) sont seulement en cours de développement et devraient permettre de résoudre le problème du stockage. Même les réservoirs devraient être construits à proximité du consommateur et moins en pleine campagne (suivant des critères de faible impact sur la nature; ce que la plupart des ouvrages de pompage-turbinage ne pourraient pas remplir).

Dans cette vision, l'énergie éolienne est un élément qui a ses limites pour pouvoir être intégrée (voir ci-dessus les 7 points sur l'énergie éolienne). Cela veut dire aussi que les éoliennes existantes puissent être démontées et ne subsistent ou ne fassent l'objet d'un 
repowering que sur les sites adaptés. Ainsi on pourra à moyen terme corriger les erreurs de développement actuelles.

En conséquence, il subsiste ou il se constitue de nouveau un cadre de vie de valeur tout en apportant une solution pour un approvisionnement énergétique respectueux de l'environnement (figure 3e). Mais pour cela il y a aussi une condition indispensable qui n'est pas représentée sur l'image: des recherches supplémentaires sur de nombreux détails et il faut beaucoup plus d'efficacité énergétique. Au moins autant que la production de courant, il faut aussi résoudre les questions de la production de chaleur et de la circulation. Pourtant, ces questions ont été jusqu'à présent à l'arrière-plan (Bachem \& Buchal 2013), alors qu'elles sont au moins aussi importantes que celle du courant électrique.

$\mathrm{Au}$ centre, il y a les questions fondamentales de l'économie et du vivre ensemble qui sont trop peu évoquées dans ce contexte. Pour réaliser un tournant énergétique véritable il faut remettre en cause les orientations de croissance actuellement prédominantes et par exemple discuter de concepts comme la suffisance (par exemple Schneidewind \& Zahrnt 2013), dont fait partie une utilisation économe et raisonnée de l'espace, tout en maintenant un certain niveau de bien-être. Comme toujours, un simple „toujours plus" est certainement en contradiction avec un véritable tournant énergétique. De tels débats de société sont multiformes et vont bien au-delà de la thématique énergétique (note 10), mais ils ont une influence sur celle-ci et sur les territoires.

Un véritable changement nécessite aussi beaucoup plus de temps que ce que le politique prévoit actuellement en Allemagne, car beaucoup de nouvelles évolutions et de nouveaux concepts doivent être mis en place. La rapidité avec laquelle on peut en principe adapter la planification et les financements comme ceux de la loi EEG, et le fait que l'on puisse démonter les éoliennes, tout cela permet d'envisager qu'une meilleure voie puisse être suivie dans un délai raisonnable.

Dans ce contexte, un nouveau mais difficile „combat pour les paysages“ vient juste de commencer avec de nombreuses ruptures menaçantes. Ce combat, on peut difficilement y échapper, même en tant que scientifique. Il exige des géographes, des défenseurs de la nature, mais aussi des techniciens, une réflexion, un positionnement et le développement de méthodes, par exemple dans le domaine de la planification et de l'intégration, de beaucoup de points de vue. Traçons ainsi le chemin vers une réconciliation et vers un tournant énergétique réussi avec une adaptation raisonnée de l'énergie éolienne, tout cela à l'échelle européenne.

\section{BIBLIOGRAPHY}

Ackermann H., Melsheimer O., Scheffran J., 2001, „Energiekonflikte. Problemübersicht und empirische Analysen zur Akzeptanz von Windkraftanlagen, Bericht eines interdisziplinären Forschungsvorhabens, Münster. Politische Verhaltensforschung 4. 
Altmoos M., Altmoos U., 2014a, Windkraft-Fakten und Natur, Internet-Publikation (deutschsprachig) unter www.freinatur.net, Nahe der Natur - Museum für Naturschutz. Link: http://www.nahe-natur.com/mediapool/88/886012/data/Windkraft-Fakten.pdf (3.Jan 2014).

Altmoos M., Altmoos U., 2014b. Blind im Wind? Augen auf: rettet unsere Landschaften vor zu vielen Windkraftanlagen. - Internet-Publikation (deutschsprachig) unter www.freinatur.net, Nahe der Natur - Museum für Naturschutz. Link: http://www.nahe-natur.com/mediapool/ 88/886012/data/Windkraft___Rettet_unsere_Landschaften.pdf (3.Jan 2014).

Bachem A., Buchal C. 2013, „Energiewende - quo vadis“, Physik Journal 12, p. 33-39.

Baerwald E.F., D’Amours G.H., Klug B.J., Barclay R.M., 2008, „, Barotrauma is a significant cause of bat fatalities at wind turbines “, Current Biology, 9/2008, 16.

BFN (Bundesamt für Naturschutz), 2011, „Windkraft über Wald“, Positionspapier, Bundesamt für Naturschutz, Bonn. p.1-8.

Coch T., Riesterer T., 2013, „Zwischen Scylla und Charybdis - wie verhält sich der Tourismus gegenüber Energiefreileitungen und Windkraftanlagen als Insignen der Energiewende“, Schriftenreihe Deutscher Rat für Landespflege, 84, p. 147-151.

Deshaies M., 2013, „Essor et limites des énergies renouvelables en Allemagne: la transition énergétique en question“, La revue de l'Energie, 613, p. 169-184.

Eichhorn M., Johst K., Seppelt R., Drechsler M., 2012, „Model-based estimation of collision risks of predatory birds with wind turbines", Ecology and Society, 17 (2).

Gailing L., Leibenath M., 2013, Neue Energielandschaften - neue Perspektiven der Landschaftsforschung, Springer, Wiesbaden, $220 \mathrm{p}$.

Hayes M.A., 2013, „Bats killed in large numbers at United States Wind Energy Facilities“, BioScience, 63, p. 975-979.

Jessel B., 1998, Landschaften als Gegenstand von Planung, Erich Schmidt Verlag, Berlin, 331p (Beiträge zur Umweltgestaltung A139).

Küster H., 1995, Geschichte der Landschaft in Mitteleuropa, C.H.Beck, München, 424 p.

Meiler M., Binder S., Faulstich M., 2011, „Speichertechnologien im Überblick“, Wasser und Abfall, 11/2011, p. 10-13.

Nägele E., 2012, „Stromerzeugung durch Windkraftanlagen - wie ändert sich die Landschaft? Versuch einer Bestandsaufnahme“, Schwäbische Heimat, 63 (3), p. 321-329.

Pierpont N., 2009, Wind Turbine Syndrom. A report on a natural experiment.

Roser F., 2013, „Ist die Schönheit der Landschaft berechenbar ?“, Naturschutz und Landschaftsplanung, 45, p. 265-270.

Rost D., 2014, Wandel (v) erkennen, Springer, Wiesbaden, 227 p.

Rydell J., Nach L., Duborg-Savage M.-J., Rodrigues L., Hedenström A, 2010, „Mortality of bats at wind turbines links to nocturnal insect migration", European Journal of Wildlife Research, 56 (6), p. 823-827.

Schaich H., Konold W., 2011, „"Moderne” und “archaische” Kulturlandschaften in Mitteleuropa“, Culterra 60 (Universität Freiburg, Institut für Landespflege), 186 p.

Schneidewind, U., Zahrnt A., 2013, Damit gutes Leben einfacher wird. Perspektiven einer Suffizienzpolitik, Ökom, München, 171p. 
Smil V., 2010, Energy Transitions, Praeger Frederick, 178 p.

Wöbse H.H., 2002, Landschaftsästhetik, E.Ulmer, Stuttgart, 304 p.

\section{NOTES}

1. sondage "conscience environnementale en Allemagne" et éoliennes (Umweltbundesamt 2012), ainsi que de nombreux autres sondages.

2. „alliance transition énergétique pour l'homme et la nature“ dans le sud-ouest de l'Allemagne, un rassemblement d'initiatives citoyennes et de scientifiques critiques, qui appellent par exemple à de nombreuses initiatives critiquant l'éolien sans remettre fondamentalement en cause la transition énergétique, mais aimeraient qu'elle soit mieux mise en œuvre: http:// www.energie-mensch-natur.de.

3. DALDORF, W., 2013. Expériences pratiques sur la rentabilité économique des parcs éoliens citoyens en Allemagne. Transparents pour le syndicat fédéral de l'énergie éolienne e. V.“ (www.wind-energie.de).

4. "le syndrome de l'éolienne“ - le terme est associé par de nombreuses thèses de science populaire avec le mal être ou même la maladie consécutive aux effets des éoliennes sur les hommes en relation avec les infrasons. Une des références est Pierpont (2009 aux Etats-Unis; voir bibliographie).

5. Le syndicat médico-social VDK a rassemblé des thèses non prouvées et de premiers indices scientifiques sur les effets des éoliennes sur la santé des hommes- un aperçu sur ce thème controversé et encore insuffisamment étudié sur le plan scientifique: http://www.vdk.de/kvkirchheimbolanden/ID139664. des faits comparables dans „Freinatur „(2014a , Literaturzitat).

6. Les firmes éoliennes et leurs syndicats ont publié différents éléments qui tendent à montrer que les accidents provenant des éoliennes peuvent être techniquement minimisés. Une synthèse par exemple dans: http://www.etz.de/files/e0s238zfe_leutron.pdf.

7. Selon les partisans de l'éolien „l'effet nocebo“, est l'affirmation (non prouvée) selon laquelle les gens se convaincraient que les éoliennes les rendent malades (et se sentiraient donc mal). Souvent abordé dans la presse et résumé dans: http://www.heise.de/tp/artikel/39/39027/1.html. 8. National Renewable Energy Laboratory (NREL 2013, USA). Des études considèrent que le barotraumatisme n'est pas valable: http://www.nrel.gov/wind/news/2013/2149.html.

9. „Studie sieht Fledermaus-Massensterben an Windrädern“ / Michael Reich (Universität Hannover): des extraits d'un projet de recherche en cours et pas encore publié et qui ont trouvé un écho dans les médias, par exemple dans SPIEGEL online, 19.08.2013. link: http:// www.spiegel.de/wissenschaft/technik/windkraft-tausende-fledermaeuse-sterben-anwindraedern-in-deutschland-a-917385.html.

10. FREINATUR, portail Internet pour la protection de la nature (www.freinatur.net), collecte d'informations sur "les énergies renouvelables, impasses et sorties“ (en allemand) par Michael Altmoos. Link: http://www.nahe-natur.com/mediapool/88/886012/data/ Regenerative_Energien___Irrwege_und_Auswege.pdf. 


\section{ABSTRACTS}

In many areas of Europe wind power is currently being developed as part of an energy revolution massively. This presents significant problems, which combines the advantages of a low-emission renewable energy more than compensate. Direct effects on human health, negative effects on biodiversity, Landschaftsverbau as a serious problem of landscape aesthetics This article presents the example of West-Germany after review of the literature and our own work on an overview of the causes of the problems and possible spatial solutions. In contrast to other dynamics of landscapes, the shoring is particularly large, abrupt, spacious and leveling effect by wind power, so that conflicts and upheavals are great. However, since wind energy such as solar energy volatile memory are needed. The energy storage but is a key issue that is not easy to release according to the present state. Given the deficit to save conventional power plants are still required, so that their environmental problems persist. As a solution, an integrative approach to spaces over the previously prevailing one-sided technical point of view is necessary so that conservation and technical opportunities are equally possible.

Dans beaucoup de régions en Europe l'énergie éolienne est développée massivement dans le cadre de la transition énergétique. Il en résulte des problèmes plus importants que les avantages que procure la production d'énergie à partir de sources renouvelables : effets directs sur la santé des êtres humains, conséquences négatives pour la biodiversité, mitage du paysage et les problèmes esthétiques qui en découlent. À partir de la bibliographie et de recherches personnelles portant sur l'exemple du sud-ouest de l'Allemagne, cet article présente une vue d'ensemble des causes, des problèmes et des solutions possibles. Par contraste avec la dynamique ordinaire des paysages, la construction d'éoliennes a des effets particulièrement importants, brutaux, étendus et dégradants pour le paysage; si bien qu'elle déclenche des conflits et des ruptures. Comme par ailleurs l'énergie éolienne est intermittente, tout comme l'énergie solaire, il est nécessaire de construire des réservoirs de stockage. Mais le stockage de l'énergie est une question clef, encore difficile à résoudre en l'état actuel de la technique. En raison du manque de réservoirs de stockage les centrales conventionnelles sont encore nécessaires; si bien que les problèmes environnementaux persistent. La solution passe par un point de vue intégrant les espaces dans leur ensemble par opposition au point de vue uniquement technicien prédominant jusqu'à présent, de façon à concilier la protection de la nature et les opportunités techniques.

In vielen Räumen Europas wird Windkraft als Teil einer Energiewende derzeit massiv ausgebaut. Daraus erwachsen bedeutende Probleme, welche die Vorteile einer abgasarmen erneuerbaren Energiegewinnung mehr als aufwiegen: Direkte Wirkungen auf die Gesundheit von Menschen, Negative Wirkungen auf Biodiversität, Landschaftsverbau als ernstes Problem der Landschaftsästhetik. Der vorliegende Artikel bietet am Beispiel Südwest-Deutschlands nach Literaturauswertung und eigener Arbeiten eine Übersicht der Ursachen, der Probleme und mögliche räumliche Lösungen an. Im Gegensatz zur sonstigen Dynamik von Landschaften ist der Verbau durch Windkraft besonders groß, abrupt, weiträumig und nivellierend wirkend, so dass Konflikte und Verwerfungen groß sind. Da allerdings Windenergie ist wie Sonnenenergie volatil, sind Speicher nötig. Die Energiespeicherung aber ist eine Schlüsselfrage, die nach gegenwärtigem Stand nicht schnell lösbar ist. Angesichts des Defizits an Speichern sind weiterhin konventionelle Kraftwerke erforderlich, so dass auch deren Umweltprobleme weiter bestehen. Als Lösung ist eine integrative Sichtweise auf Räume gegenüber der bisher vorherrschenden einseitig technischen Sichtweise notwendig, so dass Naturschutz und technische Chancen gleichermaßen möglich werden. 
INDEX

Keywords: energy transition, integrated planning, landscape impact, pump storage, wind energy Mots-clés: énergie éolienne, impact paysager, planification intégrative, réservoir de stockage, tournant énergétique

Schlüsselwörter: Energiewende, integrative Planung, Landschaftswirkung, Speicher, Windenergie

\section{AUTHOR}

\section{MICHAEL ALTMOOS}

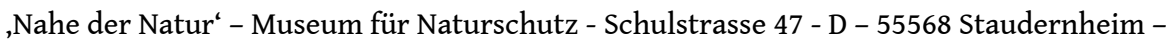
Allemagne. Internet: http://www.naturschutz-museum.de - E-Mail: info@naturschutzmuseum.de - Fon ++49-6751-8576370 - Fax ++49-6751-8576346. Biologiste (Dipl-Biol.) et géographe (Dr. rer. nat.), avec pour thèmes privilégiés la protection de la nature à travers la recherche, la pratique et la formation. Dirige le musée pour la protection de la nature de Staudernheim qui a permis la réalisation de ce travail. Il est aussi employé du Land de Rhénanie-Palatinat pour la gestion du réseau d'espaces protégés ,Natura 2000` (Directive européenne Habitats naturels). 